\title{
Effects of constitutive expression of somatolactin alpha on skin pigmentation in medaka
}

\author{
Shoji Fukamachi ${ }^{a, *}$, Takashi Yada $^{\mathrm{b}}$, Axel Meyer $^{\mathrm{a}}$, Masato Kinoshita ${ }^{\mathrm{c}}$ \\ 'Department of Biology, University of Konstanz, D-78467 Konstanz, Germany \\ ${ }^{b}$ Freshwoter Fisheries Research Division. National Research Institute of Fisheries Science, Nikko, Tochigi 321-1661, Japan \\ 'Division of Applied Biosciences, Graduate School of Agriculture, Kyoto University, Kyoto 606-8502, Japan
}

Keywords:

Somatolactin alpha (SLa)

Medaka (Oryzias latipes)

Transgenic fish

Internal ribosome entry site (IRES)

Pigment cell (chromatophore)

Lipid/cortisol metabolism

Growth-hormone receptor

\begin{abstract}
A B S T R A C T
Somatolactin alpha (SLa) is a fish-specific peptide hormone secreted from the pituitary. Its functions have been investigated for the last decade but are still under debate. We previously reported a frame-shift mutation on SLa in a medaka mutant, color interfere (ci), which shows defects in body-color regulation, lipid storage, and cortisol secretion. In this study, we examined the effects of introducing a DNA fragment which constitutively expresses wild-type SLa under regulation of the $\beta$-actin (Actb) promoter into the $c i$ genome. We successfully visualized the transgene expression by taking advantage of an internal ribosome entry site (IRES) and the green fluorescent protein (GFP). The transgenic medaka, Actb-SLa:GFP, exhibited a reversed body-color phenotype of ci; more orange xanthophores and less white leucophores. We also detected more black melanophores and less silver iridophores, which indicates SLa's comprehensive role in regulating all types of pigment cells in the skin. Unexpectedly, the defects in lipid/cortisol contents remained in Actb-SLa:GFP. Therefore, the causal relationship between SLa and lipid/cortisol metabolisms relapses to an open question which needs to be reassessed by other types of experiments. Both the ActbSLa:GFP and $\mathrm{ci}$ fish grow and mature similar to wild type, indicating SLa contributes little to growth regulation in spite of the fact that it binds to a teleost-specific paralog of growth-hormone receptor in vitro. The present study provides definitive evidence for SLa's principal and indispensable role in body-color regulation in medaka.
\end{abstract}

\section{Introduction}

Somatolactin (SL) is the closest relative of growth hormone $(\mathrm{GH})$ which is secreted from the pituitary gland of "fish" species (including lungfish). Expression of SL is up/down-regulated during various internal/external environmental changes, including sexual maturation (Rand-Weaver et al., 1992), acute stress (Rand-Weaver et al., 1993: but see Zhu and Thomas, 1995), salt/gas concentration (Kakizawa et al., 1993; but see Tang et al., 2001), season (RandWeaver et al., 1995; but see López et al., 2001), light intensity (Zhu and Thomas, 1995; but see Kakizawa et al., 1995), fasting (Company et al., 2001; but see Kakizawa et al., 1995), etc. Although SL may (or may not; as exemplified by the above conflicting evidence between species) have important functions during these circumstances, its direct effects on the body remain unknown from these observations.

Abbreviations: SLa, somatolactin alpha; SLR, somatolactin receptor; ci, color interfere; IRES, Internal ribosome entry site; Actb, $\beta$-actin.

- Corresponding author. Lehrstuhl fur Zoologie und Evolutionsbiologie, Department of Biology, University of Konstanz, D-78457 Konstanz. Cermany. Tel.: +49 753188 4066; fax: +497531883018

E-mail address: shoji.fukamachi@uni-konstanz.de (S. Fukamachi).
Several in-vitro/vivo experiments using purified/recombinant SL revealed its physiological activities in steroidogenesis (Planas et al., 1992), phosphate transport (Lu et al.. 1995), melanosome aggregation (Zhu and Thomas, 1997), phagocyte activation (Calduch-Giner et al., 1998; but see Sakai et al., 1996), lipolysis (Vega-Rubin de Celis et al., 2003), etc. However, because the effects are often weak despite the rather high doses of SL ( $>100 \mathrm{ng} / \mathrm{ml}$ ), it is still unclear whether or not endogenous SL ( $\sim 20 \mathrm{ng} / \mathrm{ml}$ in the plasma; Rand-Weaver et al. 1995; Zhu and Thomas 1996; Diez et al., 2007) actually plays significant roles in these activities.

It is important to note that some fish have two SL genes due to the teleost-specific genome duplication (Zhu et al., 2004; Fukamachi and Meyer, 2007). These genes are distinguished as SL alpha (SLa) and SL beta $(S L b)$. SLa exists across teleost species, but SLb has only been reported from relatively primitive fish such as salmon and carp (and probably also eel). Genomes of more derived species (e.g., pufferfishes and medaka) lack $S L b$, indicating that there has been a lineage-specific diversification of SL's functions. This diversification could be the cause of the conflicting evidences outlined above.

Medaka, Oryzias latipes, has only one SL gene that belongs to SLa, and a mutation (11-bp deletion) on it was previously identified in a strain called color interfere ( $c i$; Fukamachi et al., 2004). The $c i$ strain was originally isolated as a body-color mutant whose skin is 
"gray" (instead of wild-type "brown") due to a dramatic increase in both the number and size of white pigment cells (leucophores) and a concomitant decrease in orange pigment cells (xanthophores; Takeuchi, 1969). We also reported additional defects in $c i$ including increased lipids (triglycerides and cholesterols) in the liver/muscle and decreased cortisol in the plasma (Fukamachi et al., 2005). These studies suggest SLa has a pleiotropic function regulating body color, lipid storage, and cortisol secretion. These functions could be supported by the wide spread expression of $S L$ receptor $(S L R)$ in various organs, most strongly in the liver and muscle (Fukamachi et al., 2005; see also Fig. 3).

In these types of genetic studies (i.e., comparisons of a mutant and a so-called "wild-type [WT] control"), however, there are always concerns as to whether the phenotypic differences in between solely reflect the mutation of interest, or if there are significant contributions to the phenotypes from other polymorphisms/mutations in the genome (i.e., difference in genomic backgrounds). This concern needs to be paid particular attention in medaka where the genomewide SNP rate exceeds $3 \%$ between populations (Kasahara et al., 2007). $\mathrm{ci}$ is a strain that was isolated $>50$ years ago and its population of origin is unclear (Yamamoto 1975). Therefore, we planned to introduce an SLa-expression construct into the ci genome in order to examine its effects under the uniform genomic background and obtain more reliable genetic evidence for SLa's functions.

\section{Materials and methods}

\subsection{Preparation and microinjection of the SLa-expression construct}

We first PCR-amplified the following DNA fragments using overlapping primers; "a $3 \times$ FLAG tag and an internal ribosome entry site (IRES)" from the pIRES-hrGFP-1a vector (Stratagene), "a GFP open-reading frame" from the phrGFP ll-1 vector (Stratagene), and "SV40 polyA signals" from the pDsRed2-1 vector (Clontech). These fragments were joined by an additional PCR using $5^{\prime} / 3^{\prime}$ most primers, end-trimmed by Notl digestion, and cloned in to the pGEM-Teasy vector (Promega). We then amplified the medaka $\beta$ actin (Actb) promoter (including the first non-encoding exon and the first intron) by genomic PCR, which was inserted into EcoRI/Sall site of the plasmid above. Finally, we prepared an open-reading frame (ORF) of the wild-type SLa (without the stop codon) by RTPCR, and inserted the fragment into Sall/Smal site of the plasmid. We sequenced this plasmid and verified that all the fragments were properly connected.

For microinjection, we used the Notl-Noti interval of the plasmid ( $\sim 6.2 \mathrm{~kb}$; Fig. 1), which was dissolved in Yamamoto's linger solution $\left(\mathrm{NaCl} 750 \mathrm{mg}, \mathrm{KCl} 20 \mathrm{mg}\right.$, and $\mathrm{CaCl}_{2} 20 \mathrm{mg}$ in $100 \mathrm{ml}$ DDW; pH 7.3 adjusted by $\mathrm{NaHCO}_{3}$ ) at a concentration of $20 \mathrm{ng} / \mu \mathrm{l}$.

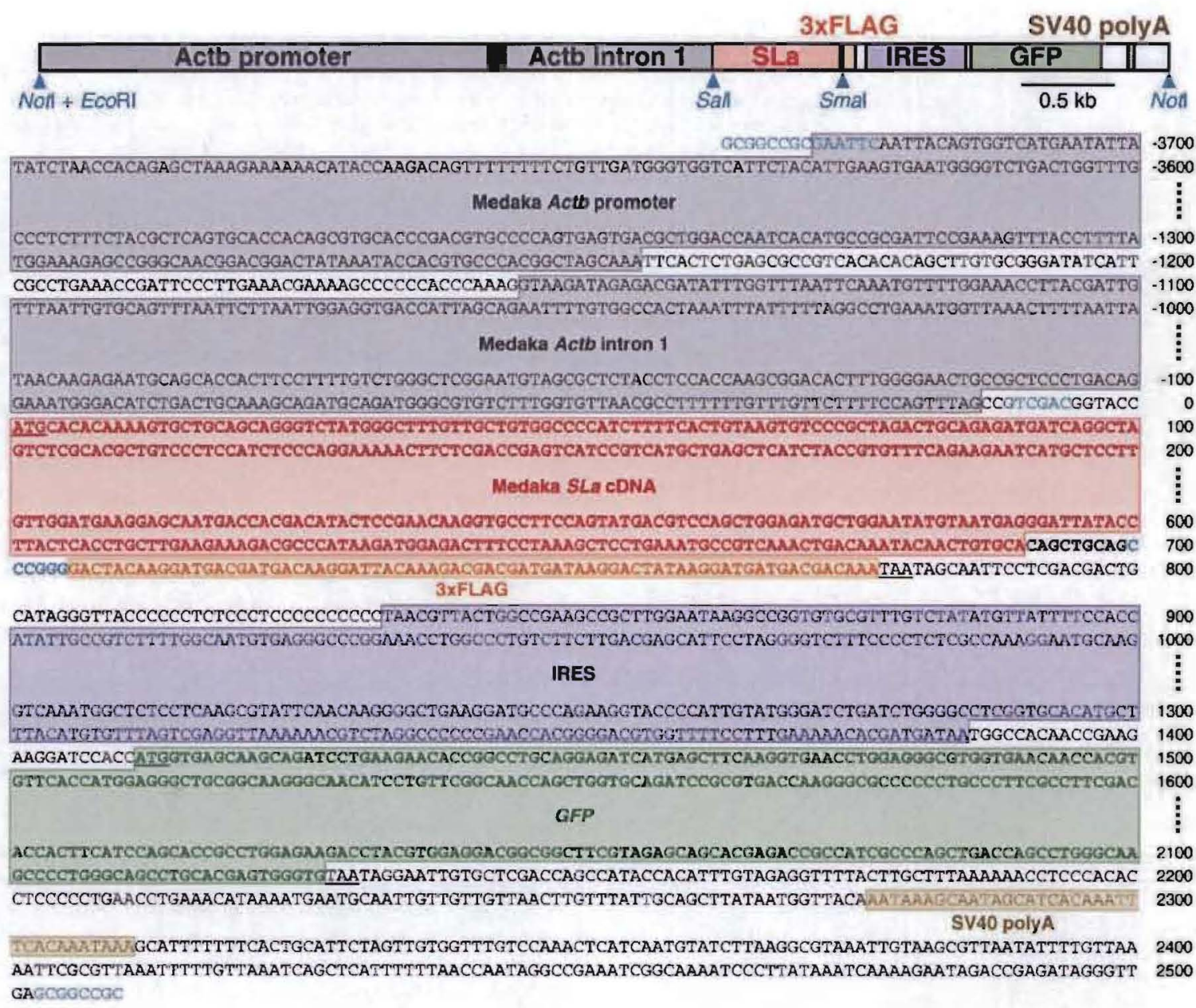

Fig. 1. The structure and partial sequence of the injection construct. The construct consists of the medaka Acth regulatory region (gray), medaka SLa cDNA (red). C-terminus $3 \times$ FLAC tag (orange), IRES (purple), ORF for GFP (green), and SV40 polyA signal (ocher). Translation start/stop codons for the two ORFs are underlined. Restriction sites used for the construct preparation are shown in light blue. (For interpretation of the references to colour in this figure legend, the reader is referred to the web version of this article.) 


\subsection{Expression of SLa mRNA from the transgene}

We isolated total RNAs from organs using Trizol (Invitrogen) and quantified them by spectrometry and gel electrophoresis. The RNAs were treated with DNase I, RNase-free (Fermentas), before we synthesized cDNAs using SuperScript III Reverse Transcriptase (Invitrogen) and polyT primer. We used PCR conditions of $94{ }^{\circ} \mathrm{C}$ for $1 \mathrm{~min} ; 20-40$ cycles of $98^{\circ} \mathrm{C}$ for $20 \mathrm{~s}, 58-60{ }^{\circ} \mathrm{C}$ for $1 \mathrm{~min}, 72^{\circ} \mathrm{C}$ for $1 \mathrm{~min} ; 72{ }^{\circ} \mathrm{C}$ for $10 \mathrm{~min}$. Products were resolved on a $1 \%$ agarose gel and photographed after ethidium bromide staining. Primer sequences are; SLa (f: $5^{\prime}$-aatggctgctgcattctgtg and r: 5'-tgcacagttgtatttgtcagtttgac), Actb (f: 5'-gattcecttgaaacgaaaagcc and r: 5'cagggctgttgaaagtctcaaac), SLR (f: 5 '-taatgatgattctcttgccacctg and r: 5'-taggaggtttcccagcaggtc), and GHR (f: 5 '-tctcctgcctcctgttcctg and $\mathrm{r}$ : 5 -gctgctcaaaaggtcaggaatc). We tried several more pairs of primers designed for different regions, with which similar results could be reproduced (data not shown).

\subsection{Quantification of body size and skin color}

We used fully mature fish of $\sim 7$ months of age. We anesthetized the fish on ice, measured body length (from the snout to the distal edge of the caudal fin) and body weight (wet body mass), and photographed the skin on the trunk (below the dorsal fin and above an anterior edge of the anal fin). We manually counted the skin pigment cells in an area of $2 \mathrm{~mm} \times 2 \mathrm{~mm}$ to calculate the density (cells $/ \mathrm{mm}^{2}$ ).

\subsection{Measurement for lipid and cortisol contents}

We used the method previously described (Fukamachi et al. 2005). Briefly, we collected $\sim 2 \mu$ of blood from the caudal vessels, obtained blood plasma by centrifugation, and measured cortisol by using Cortisol EIA Kit (Oxford Biomedical Research). We also took the liver and the muscle of the dorsal trunk from the fish, from which lipids were extracted by the chloroform/methanol method, separated by thin-layer chromatography on chromatorod SIII (Iatron Laboratories), and quantified by latroscan MK 5 flame-ionization detector (latron Laboratories).

\section{Results and discussion}

\subsection{Establishment of the SLa-transgenic ci medaka}

The ci medaka lacks 91 aa upstream of the C terminus of SLa's 230 aa due to a frame-shift mutation, and transcription of the mRNA is also greatly reduced (Fukamachi et al., 2004; see also Fig. 3). In order to express CDNA of wild-type SLa in this putative SLa-null mutant, we used the medaka 3 -actin (Actb) promoter. We did not use the native SLa promoter and other potentially essential gene-regulatory regions (e.g., comparative genomics on the SLa locus revealed an unusually highly conserved $\sim 0.4-\mathrm{kb}$ sequence in the 3 '-flanking region; data not shown), because the structure has not been well characterized and the native expression is weak under ordinary breeding conditions (i.e., fish need to be kept in dark environments to maximize the expression: Fukamachi et al., 2004). In addition, we wanted to monitor the gene expression at early stages of embryogenesis in order to quickly screen transgenic individuals. Hence, we placed an internal ribosome entry site (IRES; Fahrenkrug et al., 1999) and an open-reading frame (ORF) of green fluorescent protein (GFP) at $3^{\prime}$ downstream of the Actb-SLa (Fig. 1), so that the construct transcribes two ORFs into one mRNA from which SLa and GFP are simultaneously but separately translated (i.e., SLa is not tagged by GFP).

We microinjected the construct into 325 eggs of the $c i$ host fish, some of which successfully exhibited chimeric expression of GFP (data not shown). Although the fluorescence was much weaker than that of others such as the OIMA1-GFP strain, where GFP is placed directly under the $\alpha$-actin promoter (Kinoshita, 2004) and invisible under an ordinary fluorescent/incandescent lamp, the signal becomes obvious under a fluorescent microscope at any stage from one-day embryo to adult (Fig. 2a-d). Ninety-three of the injected $F_{0}$ fish reached adult stages, and about half of these fish exhibited marked "dark-orange" (instead of the gray of ci) skin color (Fig. 2c, e-j). Interestingly. different to the green fluorescence, the color was not chimeric, indicating that the construct was introduced into an endocrine tissue (s) (e.g., the pars intermedia of the pituitary where SLa is natively produced), which secreted functional SLa into the blood circulation to cause this color change in the whole body.

Among the dark-orange $F_{0}$ adults, we identified two females and three males that produced embryos with green fluorescence $\left(F_{1}\right)$ at
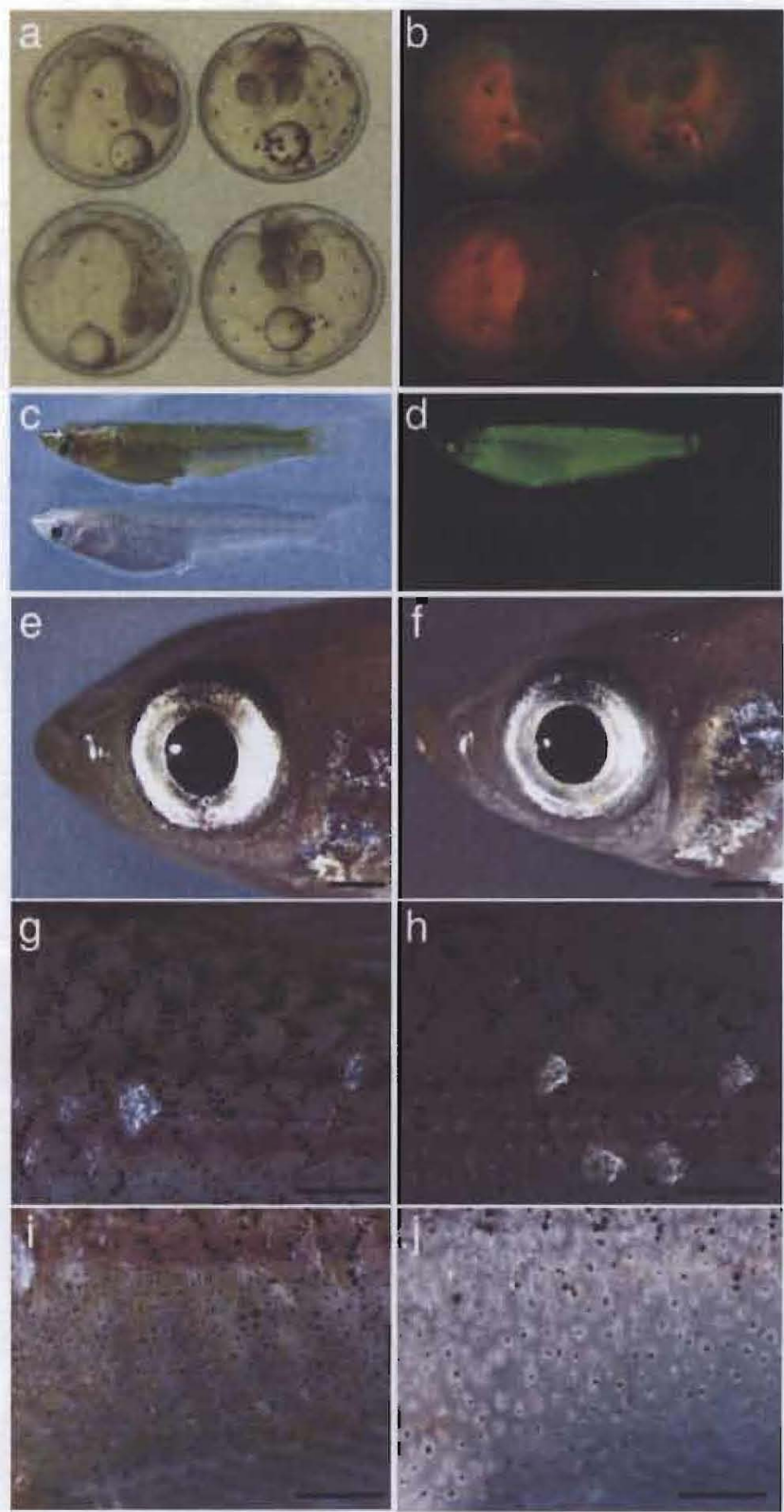

Fig. 2. Comparison of appearances between the $c i$ and Actb-SLa:GFP medaka. (a) Twoday embryos of Actb-SLa:GFP (top) and $c i$ (bottom). (b) A fluorescent image of (a). (c) Male adult fish of Actb-SLa:GFP (top) and ci (bottom). (d) A fluorescent image of $c$. (e-j) Pictures with larger magnifications of the head (e, f), dorsal trunk (g. h), and ventral trunk (i,j) of Actb-SLa:GFP (e, g, i) and $c i(f, h, j)$. Scale bars: 1 mm. 
transmission rates of $44-52 \%$ in backcrossing ( $n=216-309$ for each family), demonstrating successful germ-line integration of the expression construct. The dark-orange color of the $F_{t}$ fish becomes obvious at later stages ( $\sim 1$ month after hatch), and we verified the constitutive (ectopic) transcription of SLa by RT-PCR (Fig. 3). Based on the rule previously proposed (Kinoshita et al., 2003), this transgenic darkorange fish was termed Actb-SLa:GFP.

Thus, the IRES-GFP system under the Actb promoter was quite helpful for screening the transgenic individuals with the lateappearing phenotype (i.e., without using it, far more time and space would have been wasted raising hundreds of non-transmitters). To our knowledge, this is the first report of a functional IRES in medaka.

\subsection{Growth and sexual maturation of the SLa-transgenic ci medaka}

The aim of this study is to assess phenotypic effects of presence/ absence of SLa on uniform genomic backgrounds. Although the genomic backgrounds of $c i$ and Actb-SLa:CFP should already be highly uniform, we further backcrossed hemizygous Actb-SLa:GFP (ci/ci: $\mathrm{SLa} /-$ ) with $\mathrm{ci}(\mathrm{ci} / \mathrm{ci} ;-/-$ ) and obtained their offspring (whose genotype ratio must be ci/ci; SLa/ - : ci/ci; $-/-=1: 1$ ). These sibling fish were raised in the same tank in order to provide identical environmental conditions. We unfortunately lost many of them before they reached sexual maturation, nevertheless we obtained similar numbers of survivors; 25 gray fish ( 17 males and 8 females) and 23 dark-orange fish ( 16 males and 7 females). Both fish types swim, feed, mate, and produce dozens of fertilized eggs every morning just like WT fish do (unpublished observation) under ordinary breeding conditions (further discussed in Section 3.5).

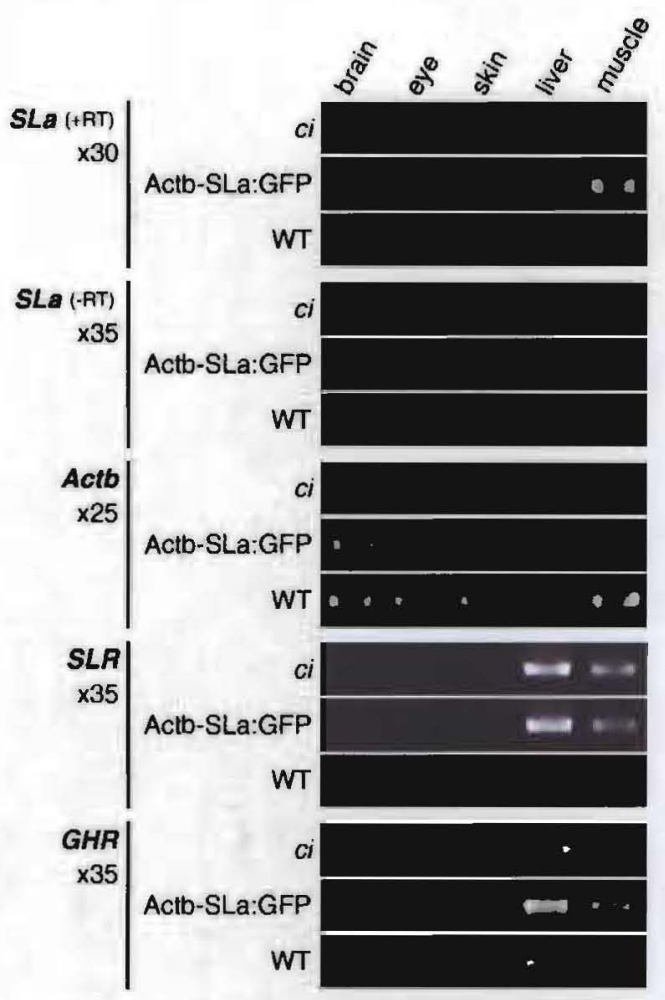

Fig. 3. Electropherograms of RT-PCR products for $S L a, A c t b$, SLR, and GHR of the $c i$, ActbSla:GFP, and WT (HB32C) medaka. The number of PCR cycles $(\times 25,30$, or 35$)$ is indicated on the left for each gene. Note that no product is detectable in the negative control (without reverse transcription: -RT) for the intron-less Sla transgene even after additional five PCR cycles. For other endogenous genes, primers are designed to sandwich an intron(s) so that products from template CDNA or contaminated genomic DNA (which was actually not detected in any reaction) can be distinguished.
Thus, neither presence nor absence of SLa should have a crucial effect on viability, growth, or reproduction in medaka.

We were particularly curious about the body size of Actb-SLa: GFP, because; 1 . SL is the closest relative of $\mathrm{CH}, 2$. SL receptor (SLR) is one of two teleost-specific duplicated $\mathrm{GH}$ receptors (GHRs; see Fukamachi and Meyer 2007), and 3. GH-transgenic medaka have dramatically (>80\%) increased body weight (Howard et al., 2004). Interestingly, however, no difference was detected in the body size (length/weight) between the matured $c i$ and Actb-SLa:GFP siblings (Fig. 4a). Therefore, even though signals from SLa and $\mathrm{GH}$ are transmitted by the cognate GHRs, their downstream cascades seem to be strictly distinguished.

\subsection{Effects of SLa on pigment cells in the skin}

As already described, an apparent effect of the SLa expression was the dark-orange color of the skin, and we quantified the pigment cells in the $c i$ and Actb-SLa:GFP siblings (Fig. 4b). A striking increase of xanthophores in Actb-SLa:GFP was obvious on both the dorsal $\left(179.3 \pm 23.6\right.$ cells $/ \mathrm{mm}^{2}$; mean \pm SEM) and ventral $\left(101.7 \pm 8.1\right.$ cells $\left./ \mathrm{mm}^{2}\right)$ sides of the trunk in comparison with those in ci $\left(34.2 \pm 4.2\right.$ cells $/ \mathrm{mm}^{2}$ and $0.3 \pm 0.2$ cells $/ \mathrm{mm}^{2}$, respectively). In contrast, leucophores are decreased in Actb-SLa:GFP on the dorsal side of the trunk $\left(1.2 \pm 0.3\right.$ cells $/ \mathrm{mm}^{2}$ in Actb-SLa:GFP and $12.3 \pm 0.6$ cells $/ \mathrm{mm}^{2}$ in ci). Ventral leucophores do not differ in number $\left(23.6 \pm 3.2\right.$ cells $/ \mathrm{mm}^{2}$ in Actb-SLa:GFP and $20.3 \pm 1.2$ cells/ $\mathrm{mm}^{2}$ in ci), but their size (dendritic cell shape) is apparently reduced in Actb-SLa:GFP (Fig. $2 \mathrm{i}$ and $\mathrm{j}$ ). The symmetrical phenotypes in the SLa-absent ( $c i$ ) and SLa-present (Actb-SLa:GFP) strains produced under uniform genomic backgrounds demonstrate the indispensable role of the medaka SLa in enhancing differentiation of xanthophores and suppressing differentiation of leucophores.

Additional effects of the transgene were detected in other types of pigment cells in the skin; silver iridophores and black melanophores. The abdomen and the opercle of Actb-SLa:GFP looked less iridescent in comparison with those of $c i$ (Fig. 2c, e, and f), indicating that iridophore differentiation is suppressed by SLa. By contrast, differentiation of the melanophores is enhanced in Actb-SLa: GFP (dorsum, $100.1 \pm 10.7$ cells $/ \mathrm{mm}^{2}$; venter, $87.3 \pm 6.4$ cells $/ \mathrm{mm}^{2}$ ) in comparison with that in ci (dorsum, $39.6 \pm 1.3$ cells $/ \mathrm{mm}^{2}$; venter, $18.2 \pm 1.0$ cells $/ \mathrm{mm}^{2}$; Fig. $4 \mathrm{~b}$ ). In previous comparisons between ci and WT, we did not detect such iridophore/melanophore differences (Fukamachi et al. 2004; 2006). Therefore, the iridophore/ melanophore phenotypes could be a side effect of ectopic overexpression (Fig. 3) and might not occur in natural conditions. However, the present result indicates that SLa may instigate physiological activities which increase the number of light-absorbing pigment cells (xanthophores/melanophores) and decrease the light-reflecting cells (leucophores/iridophores) that can facilitate harmonious body-color changes.

\subsection{Effects of SLa on lipid storage and cortisol secretion}

We expected the SLa transgene to also rescue the non-pigmentation anomalies in $c i$, therefore we measured triglyceride contents in the liver/muscle and cortisol concentration in the plasma of Actb-SLa: GFP and ci. However, we unexpectedly detected no significant difference in triglyceride nor cortisol contents (Fig. 5). When we measured these values in WT, we consistently observed less triglycerides and more cortisol than we did in ci (Fig. 5), even though the present WT (Southern Sakura) is genomically divergent from the previous one (Northern HNI). Hence, although $c i$ actually has defects in lipid/cortisol metabolisms and functional SLa is successfully expressed in Actb-SLa:GFP according to the GFP expression and the skin color (Fig. 2), these defects could not be rescued, with the exception of the fatty liver being significantly reduced in size (Fig. 5). 
a

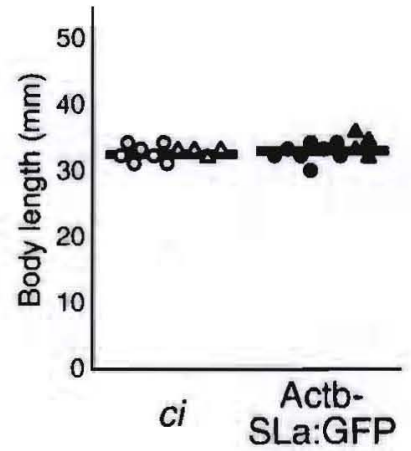

b

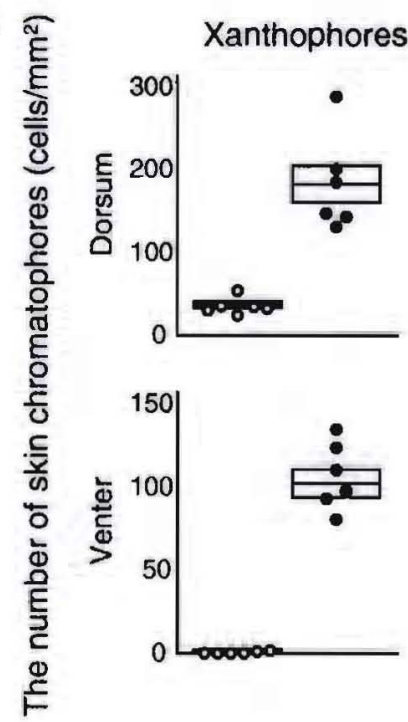

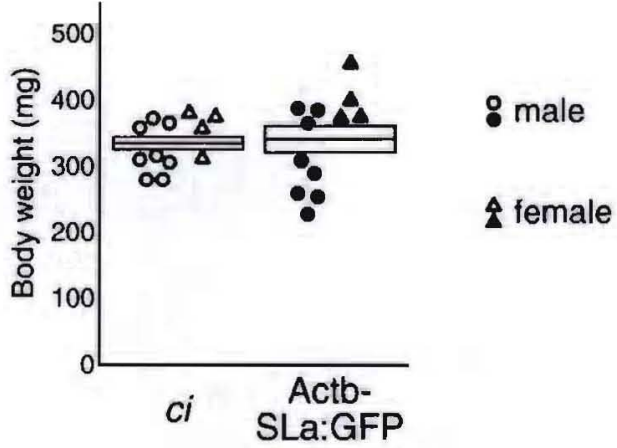

Leucophores

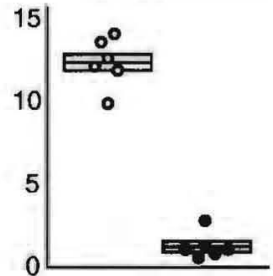

Melanophores
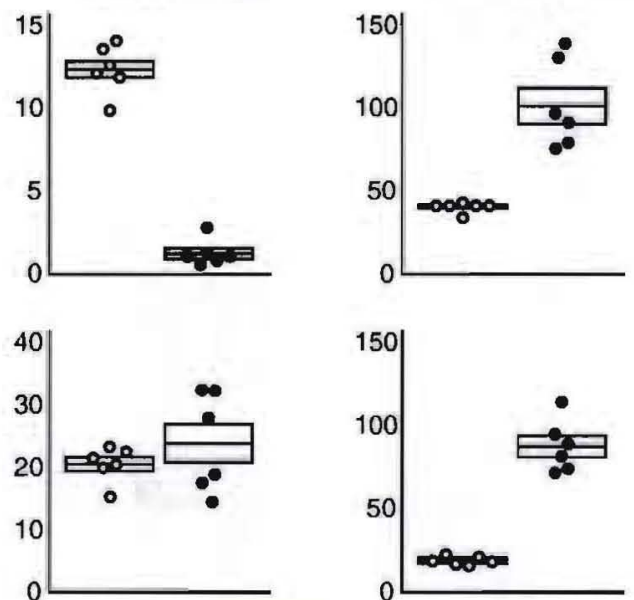

- ci Actb-SLa:GFP

Fig. 4. Quantification of body size and skin color of $c$ and Actb-SLa:GFP. (a) The body size. Length and weight of fully matured males (circles) and females (triangles) were measured. No significant difference was detected ( $P=0.368$ or 0.790 , respectively; Student's two-tailed $t$-test). (b) The skin color. The numbers of visible xanthophores and melanophores in Actb-SLa:GFP are significantly increased in both the dorsal and ventral sides of the trunk $(P<0.001$; Student's two-tailed $t$-test). Leucophores are significantly decreased only in the dorsal trunk (dorsum, $P<0.001$; venter, $P=0.348$; Student's two-tailed $t$-test).

Interpretation of these results is difficult, but there are a few possible explanations. One is that the lipid/cortisol anomalies in ci are not caused by the mutation on SLa but reflect different genomic backgrounds (see Introduction). Such putative polymorphisms/ mutations seem to be unique in the ci genome, because lipid/cortisol contents are consistent between the highly divergent Northern/ Southern WT fishes (Fig. 5; Fukamachi et al. 2005). However, this explanation is unlikely, because SL-lipid correlation has been suggested in multiple fish species (Company et al., 2001; Yada et al., 2002; Vega-Rubin de Celis et al., 2003) and SLR is most strongly expressed in the liver and muscles where lipids are accumulated in $\mathrm{Ci}$ (Fukamachi et al., 2005).

An alternative explanation is that all the defects in $\mathrm{ci}$ are indeed caused by the mutation on SLa, but only part of the defects were rescued by the method adopted in this study: SLa expression by the Actb promoter (Fig. 1). Generally, hormones activate receptors in a dose-dependent manner, and a dose-response curve of SLa has been known to become bell shaped (Calduch-Giner et al., 1998); i.e., over-dosing inhibits activation (dimerization) of the receptor. We found that SIR (and also GHR) is transcribed at a similar level between $c i$ and Actb-SLa:GFP in all organs examined (Fig. 3), and, based on the rescued skin color (Figs. 2 and 4b), we have no reason to believe that the expression of SLa in Actb-SLa: GFP is too low to activate the receptor. We speculate that the extracellular concentration of SLa in these non-rescued target organs could be too high (due to ectopic para/autocrine actions?), but this question needs to be addressed by further experiments, such as quantitative trait loci (QTL) mapping of the lipid/cortisol phenotypes, purification and dosing of recombinant SLa protein to ci, additional transgenic ci strains using series of weaker/native promoters, etc.

\subsection{Future use of the ci and Actb-SLa:GFP strains}

We established the Actb-SLa:GFP strain in order to provide a useful framework for future studies. In theory, any phenotypic differences between the Actb-SLa:GFP and ci medaka must depend solely on the presence or absence of the single transgene (Fig. 1), which should immediately provide direct and conclusive evidence for SLa's functions. Even though the difference could be a side effect of the constitutive/ectopic expression, and may not occur in natural conditions, the results show SLa's physiological activities in vivo. Although we revealed differences only in skin color (Fig. 4b) and liver size (Fig. 5), additional differences may become obvious when fish are kept under specific conditions. For example, if SLa plays an essential role in stress/immune response or osmoregulation (see Introduction), either Actb-SLa:GFP or ci would show a defect (e.g., higher/lower mortality) when the fish are infected by pathogens or put in salt water. 

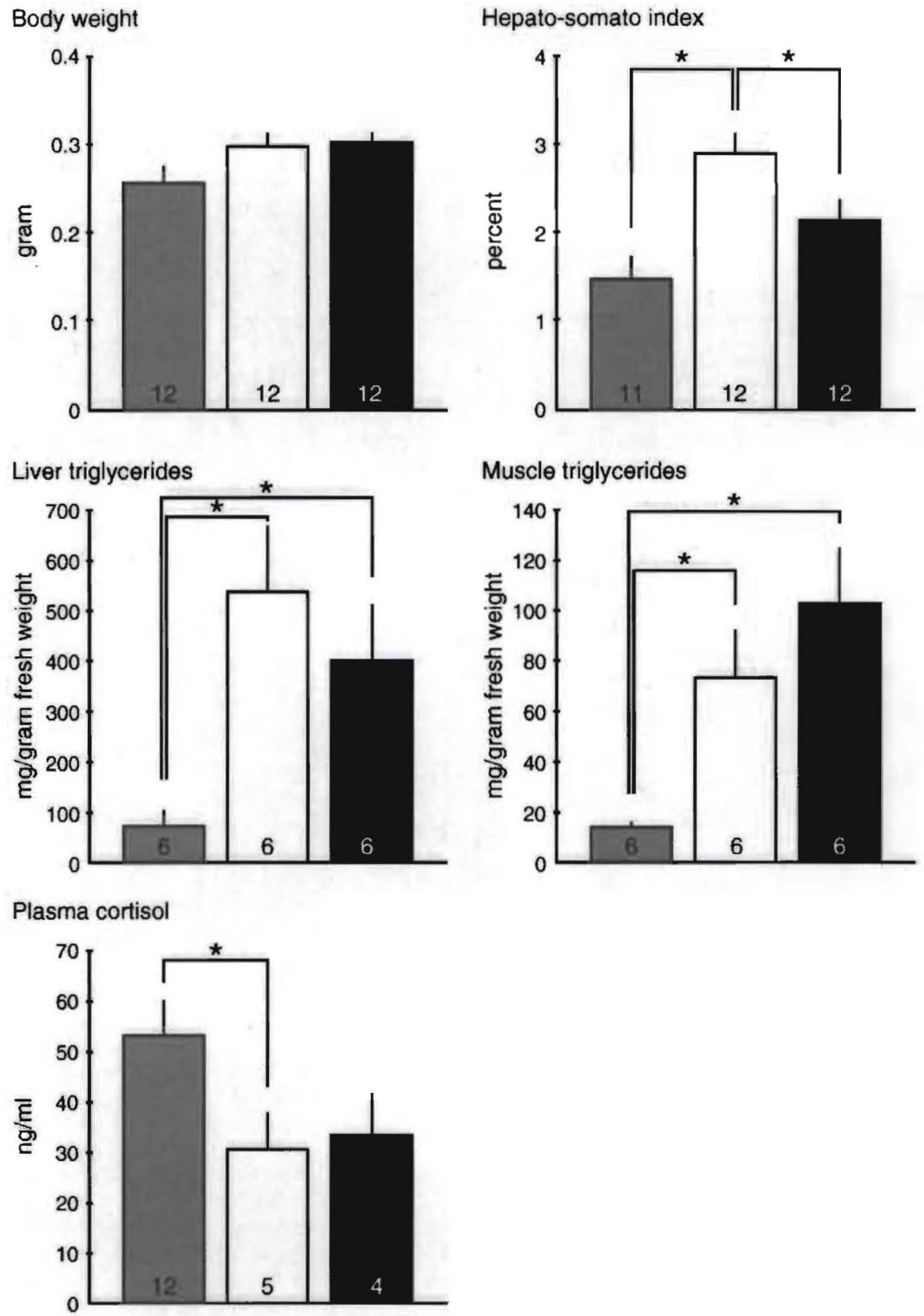

Fig. 5. Lipid and cortisol contents of WT (Sakura; gray), ci (white), and Actb-SLa:GFP (black). Results are shown as means \pm SEM. Sample sizes are indicated in each bar. Data was analyzed by ANOVA followed by Student's $t$-test or Mann-Whitney $U$-test. *:P<0,05.

We believe that Actb-SLa:GFP and $c i$ with minimal genomic background differences should be useful for screening downstream targets of SLa in experiments such as subtraction or microarray. Although questions will remain when Actb-SLa:GFP and ci exhibit asymmetric defects (as discussed for the lipid/cortisol phenotypes in Section 3.4), these medaka tools will provide further unique opportunities for studying SLa in vivo.

\section{Acknowledgments}

The authors thank S. Oda and H. Mitani of the University of Tokyo for providing the SLa CDNA, M. Schartl of University of Würzburg for providing the $\mathrm{HB} 32 \mathrm{C}$ medaka, and J.Jones of the University of Konstanz for helpful comments on the manuscript. This work was supported by a Long-term Fellowship of the International Human Frontier Science Program Organization to S. F. (\#00059/2005-L), and grants from the Deutsche Forschungsgemeinschaft and the University of Konstanz to A. M.

\section{References}

Calduch-Giner, J.A, Pendon, C. Vaidivia, MM, Perez-Sanchez J 1998 Recombinant somatolactin as a stable and bioactive protein in a cell culture bioassay: development and validation of a sensitive and reproducible radioimmunoassay. J. Endocrinol. 156, 441-447.

Company, R., Astola, A., Pendon, C., Valdivia, M.M., Perez-Sanchez, J., 2001. Somatotropic regulation of fish growth and adiposity: growth hormone (GH) and somatolactin (SL) relationship. Comp. Biochem. Physiol., C Toxicol. Pharmacol. 130, 435-445. 
Diez, A., Menoyo, D., Pérez-Benavente, S., Calduch-Giner, J.A., Vega-Rubin de Celis, S. et al., 2007. Conjugated linoleic acid affects lipid composition, metabolism, and gene expression in gilthead sea bream (Sparus aurata L). J. Nutr. Jun. 137 gene expre $1363-1369$.

Fahrenkrug, S.C., Clark, K.J., Dahlquist, M.O., Hackett Jr., P.B., 1999. Dicistronic gene expression in developing zebrafish. Mar. Biotechnol. (NY) 1. 552-561.

Fukamachi, S. Meyer, A., 2007. Evolution of receptors for growth hormone and somatolactin in fish and land vertebrates: lessons from the lungfish and sturgeon orthologues. J. Mol, Evol. 65, 359-372.

Fukamachi, S., Sugimoto, M., Mitani, H., Shima, A., 2004. Somatolactin selectively regulates proliferation and morphogenesis of neural-crest derived pigment cells in medaka. Proc. Natl. Acad. Sci. U. S. A. 101, 1066I-10666.

Fukamachi, S., Yada, I., Mitani, H., 2005. Medaka receptors for somatolactin and growth hormone: phylogenetic paradox among fish growth hormone receptors. Genetics 171. 1875-1883.

Fukamactii, S. Wakamatsu, Y., Mitani, H., 2006. Medaka double mutants for color interfere and leucophore free: characterization of the xanthophore-somatolactin relationship using the leucophore free gene. Dev. Genes Evol, 216, 152-157.

Howard, R.D., DeWoody, J.A.. Muir, W.M., 2004. Transgenic male mating advantage provides opportunity for Trojan gene effect in a fish. Proc. Natl. Acad. Sci. U. S. A. 101. 2934-2938.

Kakizawa, S., Kaneko, T., Hasegawa, S., Hirano, T., 1993. Activation of somatolactin cells in the pituitary of the rainbow trout Oncorhynchus mykiss by low environmenta calcium. Gen. Comp. Endocrinol. 91, 298-306.

Kakizawa, S., Kaneko, T., Hasegawa, S., Hirano, T., 1995. Effects of feeding, fasting background adaptation, acute stress, and exhaustive exercise on the plasma somatolactin concentrations in rainbow trout. Gen. Comp. Endocrinol. 98, 137-146.

Kasahara, M., Naruse, K., Sasaki, S., Nakatani, Y., Qu, W., et al., 2007. The medaka draft genome and insights into vertebrate genome evolution. Nature 447, 714-719.

Kinoshita, M., 2004. Transgenic medaka with brilliant fluorescence in skeletal muscle under normal ligbt, Fish. Sci. 70, 645-649.

Kinoshita, M. Yamauchi, M., Sasanuma, M., Ishikawa, Y., Osada, T., et al., 2003. A transgene and its expression profile are stably transmitted to offspring in transgenic medaka generated by the particle gun method. Zool. Sci. 20, 869-875

López, M., Figueroa, J., Kausel, G., Vera, M.I., Krauskopf, M.. 2001. In siru hybridization of somatolactin transcripts in the pituitary glands from acclimatized carp (Cyprinus carpio). Biol. Res. 34, 7-13.

Lu, M. Swanson, P., Renfro, J.L., 1995. Effect of somatolactin and related hormones on phosphate transport by flounder renal tubule primary cultures. Am. J. Physiol. 268, R577-R582.
Plands, J.V., Swanson, P., Rand-Weaver, M., Dickhoff, W.W., 1992. Somatolactin stimulates in vitro gonadal steroidogenesis in coho salmon, Oncorhynchus kisutch. Gen. Comp. Endocrinol. 87, I -5.

Rand-Weaver, M., Swanson, P., Kawauchi, H., Dickhoff, W.W., 1992. Somatolacrin, a nove pituitary protein: purification and plasma levels during reproductive maturation of coho salmon. J. Endocrinol. 133, 393-403.

Rand-Weaver, M., Pottinger, T.G.. Sumpter, J.P., 1993. P'asma somatolactin concentrations in salmonid fish are elevated by stress. J. Endocrinol. 138, 509-515.

Kand-Weaver, M., Pottinger, T.G.. Sumpter, J.P., 1995. Pronounced seasonal rhythms in plasma somatolactin leveis in rainbow trout. J. Endocrinol. 146, 113-119.

Sakai, M., Kobayashi, M.. Kawauchi, $H_{n}$ 1996. In vitro activation of fish phagocytic cell by $\mathrm{GH}$, prolactin and somatolactin. J. Endocrinol. 15I, 113-118.

Tang, Y. Shepherd, B.S., Nichols, A.l., Dunham, R., Chen, T.T., 2001. Influence of environmental salinity on messenger RNA levels of growth hormone, prolactin, and somatolactin in pituitary of the channel catlish (/ctalurus punctarus). Mar. Biotechnol. (NY) 3. 205-217.

Takeuchi. T., 1969. A study of the genes in the gray medaka, Oryzias tatipes, in reference to body color. Biol. J. Okayama Univ. 15, 1-24.

Vega-Rubin de Celis, S., Gómez, P., Calduch-Giner, J.A., Médale, F., Pérez-Sánchez, J. 2003. Expression and characterization of European sea bass (Dicentrarchus labrax) somatolactin: assessment of in vivo metabolic effects. Mar. Biotechnol. (NY) 5 $92-101$.

Yada, T., Moriyama, S., Suzuki, Y., Azuma, T., Takahashi, A., et al., 2002. Relationships between obesity and metabolic hormones in the "cobalt" variant of rainbow trout. Gen. Comp. Endocrinol. 128, 36-43.

Yamamoto. T., 1975. An outline of the genetics of the medaka. In: Yamamoto, T. (Ed.). MEDAKA (killifish): Biology and Strains. Keigaku Pub. Co., Tokyo, pp. $154-169$.

Zhu, Y., Thomas, P., 1995. Red drum somatolactin: development of a homologous radioimmunoassay and plasma levels after exposure to stressors or various backgrounds. Gen. Comp. Endocrinol, 99, 275-288.

Zhu, Y., Thomas, P'., 1996. Elevations of somatolactin in plasma and pituitaries and increased alpha-MSH cell activity in red drum exposed to black background and decreased illumination. Gen. Comp. Endocrinol. 101, 21-31.

Zhu, Y.. Thomas, P., 1997, Effects of somatolactin on melanosome aggregation in the melanophores of red drum (Sciaenops ocellatus) scales. Gen. Comp. Endocrinol. 105. $127-133$.

Zhu, Y., Stiller, J.W., Shaner. M.P., Baldini, A., Scemama, J.L., Capehart, A.A., 2004. Cloning of somatolactin alpha and beta cDNAs in zebrafish and phylogenetic analysis of two distinct somatolactin subtypes in fish. J. Endocrinot. 182, 509-518. 